\title{
Simple Technology is an Improved Solution for a Post- Pandemic Informative System: A Reference Model
}

\author{
https://doi.org/10.3991/ijet.v16i16.23211
}

\author{
Mohamed Housni( $\left.{ }^{\bowtie}\right)$, Mohammed Talbi, Abdelwahed Namir \\ Hassan II University of Casablanca, Morocco \\ mohamed.housni.etu@etu.univh2c.ma
}

\begin{abstract}
COVID-19 pandemic accelerated the digitalization and the implementation of technological tools to distribute knowledge and content to certify the instructional process's steadiness despite the restrictions posed in many nations worldwide. However, multi-models of development and integrations based on multitudes of theoretical and conceptual frameworks made it difficult for deciders during the year - especially in developing countries - to follow a clear path based on their contextual needs. Based on a literature review and historical Data, Learning Analytics research, and its empirical results, this article proposes a dataanalytics model for growth. Training/educational technologies help stakeholders use data as intelligence sources to implement technologies that will improve traditional learning procedures without constraining practices. As a result, the paper also suggests, according to the pragmatic results, a 4-year plan applicable in multidimensional contexts to enhance the organization's learning capabilities as a whole unit to face future trials. The return on experience in the last year of the pandemic contracts the methodological basis of results. Furthermore, this manuscript's aim is defined by the urgent necessity of post-pandemic solutions to positively safeguard the future of keeping the wheel of knowledge running for the learners and warrant upcoming transition into using data as a source of developing new learning technologies.
\end{abstract}

Keywords — covid-19, digitalization, analytics, learning, decision-making

\section{$1 \quad$ Introduction}

The covid-19 pandemic has expectedly impacted the technological sector, specifically developing tools to distribute educational content synchronously (live) and asynchronous fashion (recorded). As the digitalization of learning become more relevant than ever, its efficiency and effectiveness rose to the question, not to dismiss it but to improve integration into a hybrid environment [1].

Hybrid environment definition relates to organizational understanding. However, all definitions converge into a mixture of traditional methods of instruction tools and the inclusion of advanced digital methods and instruments to distribute and manage learning content. Hybrid environments also congregate towards the augmentation of digital spaces into osmosis of physical and digital spaces [1]. 
In this environment, Data captured using digital sensors. Data analytics is the practice of extracting intelligence from data, and it became more relevant in education as "Learning Analytics.". Learning Analytics emerges as a field of study that uses Data as a driver to enhance teaching practices and ease the learning process's comprehension with consideration to contextual events. It is highly rooted in the connectivism theory of learning. Furthermore, its research study flourished distinction from qualitative educational models into quantitative, data-based models [1].

In this manuscript, an overlook of the recent Learning Analytics (L.A.) advances structure the model conceptualization and construction basis [1]. In contrast, to develop a new reference model for post-pandemic educational technologies that integrate databased systems into the educational system, applying and combining recent empirical evidence suggests new links to solve recent paradigms issues.

\subsection{The impact of covid-19 on the technological landscape}

The patterns that emerged of the virtualization options showed a humbling loss of confidence in high-end learning solutions. The communities of teachers, learners, and administration find significant resistance and difficulties to adapt to the new normal and took the luxury of well-planned and arranged transitions [2].

Digital tools become an obvious solution to this problem as physical space is not needed. However, a silent perception steers the idea that the digital spaces are unlimited to support large sizes of learners and can sustain the incredible growth in demand for it without restrictions [2].

As the recent events show, limitations in graphics cards and power supplies units, and other materials, was due to the high demand from emerging markets, like the cryptocurrency and individual gaming units. It becomes evident that the digital spaces undergo constraints by the capacity to answer global market demands. Because of the covid-19 impact, other industries that produce the materials needed for digitalization have suffered from the restrictions imposed, and the travel regulations implemented [2].

\subsection{Literature review and problem statement}

Learning Analytics state of the art alludes to the estimation, assortment, examination, and revealing of instruction using an enormous dataset relevant in time and space of learning practices. Its purpose aims to comprehend the elements of the hybrid environment in which learners learn and understand the climate where teaching occurs, besides enhancing the organization's choices of development and anticipate results dependent on contextual events such as the covid-19 pandemic. Numerous models, programs, software, equipment, and hardware have subsequently been used and exploited by the instructional organization to confront connotations of anticipated or unanticipated events [3].

Even if most of the studies refer to the second conference on Learning Analytics and Knowledge in 2012 (LAK2), most major MOOC platforms (Massive Open Online Course) release in the same year. L.A.'s definition as: "the measurement, collection, 
analysis of data about learners and their contexts" still holds weight. However, Learning Analytics research finds its roots in the 2010s $4^{\text {th }}$ International Multi-Conference on Society, Cybernetics, and Informatics, where Learning Analytics definition as a tool to support educational institutions' business aspects. 2016 marks a significant milestone in Learning Analytics research as it becomes more evident that data can steer behaviors and votes of the population. Research in Computer Science and Learning Analytics field has doubled since then. The academic conversations of analytics also become keener on taking advantage of data [3].

As the end of 2020, basic research in all referenced research databases in Web of Knowledge search-engine about Learning Analytics in general sciences, the results return a waving of more than 3300 manuscripts. $49 \%$ of the published manuscripts are articles, and another $49 \%$ are meeting papers. Only $2 \%$ of the published papers embody a review nature. The share of papers published in the computer science field represents $59 \%$. Furthermore, only $20 \%$ of research is related to engineering and mathematics. In sorting, 1952 papers show up, 63\% are meetings and conferences papers, 37\% are scientific articles, and $2 \%$ represent review papers of the topic in computer science. One noticeable remark is that the Learning Analytics field crosses between Technology science and advances, Social Science, and Humanities [3]. The United States of America gets the top place in research results with a $22.5 \%$ count of the total publication size. However, the European union - with inclusion to the United Kingdom - produced a total of more than $70 \%$, in which the leading majority goes to Spain $(18.4 \%)$, the United Kingdom (15.2\%) and Germany (10.6\%), China also has a strong position in the field with $14 \%$ research cut followed by Australia with $11.5 \%$. Nevertheless, Other countries led by Canada produced a significant amount of research that ranges between $2 \%$ and $6.5 \%$. Research results show that the leading countries in Learning Analytics are the USA, Spain, United Kingdom, China, Germany, Australia, and Canada. Moreover, developing countries have a noticeable fading, which explains the trend of applying highlevel data systems based on ready-to-use hosting services [3].

In shorts, the literature shows an increase in analytics in an educational setup and environment. In the beginning, L.A. was considered a tool for academic effectiveness (instructional aspects to support students) and support for finance operations and marketing (business aspects). L.A.'s roots share a common goal with this paper that articulates a plan to use data to support the instructional organization's development. In perspective, the results refer to six dimensions of development of technological tools [4] that adhere to LA:

1. Deciders: Stakeholders (profit from outcomes) and Clients (use the outcomes)

2. Objectives: Reflective (Self-improvement) and Predictive (Future improvements)

3. Data: Open (Available) and Restrictive (Not available)

4. Internal systems: Teams (Groups with shared goals) and Skills (Capacities to solve complex problems)

5. External systems: Norms (Cultural and social regulations) and Entourage (Contextual environment factors)

6. Tools: Instruments (Technological devices for analytics) and Methods (Techniques, approaches, practices, and procedures to achieve analytics) 
As described, L.A. dimensions do not include pedagogical aspects. As a result, it is a pedagogically neutral process that does not support or ignore a specific theoretical framework of learning or teaching. However, some MOOC technologies implement Analytics to adhere to a specific pedagogical framework or inspired by them [4].

Following the years 2013, 2014, 2015, until 2016, more findings suggest a specific framework of analytics inside MOOCs that should promote seven vectors [4] of development:

1. Goal setting: user's clear and specific aims related to a timeframe can be measured and achieved in their user dashboards.

2. Planification: strategy to schedule, design, and distribute course content that fits the learner's technologies and contextual elements.

3. Self-evaluation: Feedback to learners to show the level of closing the gap of understanding the course materials and tools.

4. Tasks: homework strategy of the course where learners are encouraged to achieve approachable sub-goals.

5. Elaboration: Combining prior historical knowledge to newly constructed one with meaning related to the learners' fundamental truths.

6. Help: support systems that are accessible and return positive feedback when used to reinforce help-seeking.

7. Certification: Rewards to the efforts and recognition of acquired knowledge.

At the beginning of 2017, more vectors introduction followed the 2016 United States of America elections' historical events and The Brexit results. Data Analytics was and still is considered a weapon of shifting behavior and policies of individuals and groups. Furthermore, since then, more consideration of analytics's ethical aspects has been studied to push analytics to positively impact users' critical thinking and encourage them to acquire facts and knowledge outside of their digital social bubble [4].

Generally, predictive models and methods are the dominating norms since the birth of the L.A. field. However, since 2016, predictive models have decreased considerably due to the shift towards relationship mining methods and the distillation of human judgment and behavior. This shift comes to consideration because the focus on retention and grade predictions is more linked to the student experience than a course variable. L.A. definition deepening the understanding of students learning experiences [4].

L.A.'s characterization as an interdisciplinary field where Computer Scientists, Learning Scientists, Psychometricians, and Researchers from other fields share the same goal to understand and improve learning using Big Data and Educational Machines and Technology started in 1954 with Skinner. L.A. literature argues that researchers and practitioners should review the analysis's acts that orient toward understanding the learning environment and not be closed to interdisciplinary crossovers to understand issues and solve them to build upon this technological leap. L.A. could contribute more if the ethical issues were solved [4]. 
Paper-Simple Technology is an Improved Solution for a Post-Pandemic Informative System: A Ref...

\subsection{Gap and contribution}

The rise of MOOCs, Technology Enhanced Learning (TEL), and Educational Data Mining (EDM) pushed forward Learning Analytics research in the last decade. Nevertheless, a lack of studies does not address Learning Analytics' application in a context where restrictions impose [5].

This paper anticipates contributing to the previous studies with the inclusion of the current situation to become the basis for future research that studies the model of technology implementations inside the educational institutions that suffer the urgency of digitalization. It is also essential to address the technological model applications made during the pandemic with a critical eye to achieve a meta-analysis of the practices during difficulties. However, this study tries to plan pathways for future technologies that include more dimensions and vectors. Proponents of the race towards using data to bring change and adapt to new era challenges this research find stable ground that encourages the study of Learning Analytics application on a hybrid educational environment to adapt and improve the models used in many contexts [5].

\section{$1.4 \quad$ Research questions and hypotheses}

In perspective, founded on the ontological/epistemological positions. Truth implicit, not singular to a context, and Data is the way to comprehend it. The quest to research the future truths about the development of new technologies facilitates learning and acquiring knowledge and information. Based on developing an analytical model and strategy to support future endeavors [6]. This inquiry attempts to respond to how Learning Analytics empirical evidence allows practitioners to develop new educational technologies that can face the everchanging environment in a fixed practice model of teaching and learning?

In this outlook, this paper poses two hypnotical results:

- H1: The ferocious hybridization of physical and digital instructional environment caused by health, economic, and social crises. Impose forward strategical thinking on administration and stakeholders to use data as a source of future knowledge about the challenges laid ahead. However, the fear of change and traditions causes malpractice and mismanagement of the strategic plans. Therefore, by simplifying processes and base decisions on data, organizations can reduce the adverse effects and expand the lifecycle of performances in educational technologies.

- H0: More technological advancements in developing educational technologies are not required. Therefore, future endeavors require only producing models that include more of existing technology with effective implementation models.

\section{Methods}

In the investigation, this paper follows a general morphological analysis (GMA). Our method is designated to recommend a systemic model structure to solve the current issue based on empirical evidence in the literature-besides, it simulates the possible 
results to offer a multidimensional analysis for a not quantifiable and complex problem [7].

The method followed in this theory-testing/exploratory paper, influenced by a collection of related concepts that suppose learning is defined by acquiring information and using it to solve contextual problems that transformed into practical knowledge [7].

Also, this paper supposes the continuation of learning, and that is not restricted to environmental factors. It is a continuous process and a human need that should answer constantly. In this sense, the paper method is guided by the belief that learning happens while using technological communication and information tools. Therefore, the obligation to guide this process no matter to the internal, individual processes of learning [8]. The trending theme of model construction is to avoid the linkage of learning with variables that may classify certain people and augment prejudice arguments. The belief of this study resides in the ability to learn is shared between all human beings. The methodology approach to answers the study inquiry, a numerical simulation was built based on one goal: to ensure a high probability success rate in the knowledge evaluation of students considering their pace of acquiring data and transforming it into information [8].

The first primordial simulation description supposes that a learner passes an evaluation at the end of each week with 20 questions. Ten of them are optional, while the others are not. The first ten questions investigate the acquisition of 10 essential data points presented at the beginning of the week and discussed in the course materials during the week from different perspectives to transform them into information. The ten optional questions are designed to connect future information during upcoming weeks that is not disclosed during the current week.

The acquisition of the 10 data points allows the learner to pass to the next week to collect more data and transform it into information related to their context. In this sense, the learner level up by 1 degree and pass to the next week if they can acquire ten information. If the learner acquires more than the essential 10 points and answers the noncompulsory questions, they can pass on the weeks.

For illustration, if the learner answers the first week, ten basic questions plus one optional, they can level up by 2, which means that they have passed the following week's requirements and can directly access the $2^{\text {nd }}$ and $3^{\text {rd }}$-week content.

The process repeats until ten weeks. The learner cannot level up past 10 degrees a week cause of the higher complexity level ahead for traditional educational content. The range of student's scores goes from 0 to 20 . If the learner scores a value inferior to 5 , then he goes back a degree and levels down to the past week. On the other hand, if the learner acquires a score value between or equal to 5 to 10 , the learner can pass to the following week. This condition is meant to ease access to further progress.

Additionally, if the learner acquires more than ten scores, the learner moves up " $n$ " weeks up to 10 weeks. Furthermore, to compensate for the situations where learners restart their progress, the value of the total number of students is fixed to $10 \%$ but can be changed in the simulation for future experiences. 
Paper-Simple Technology is an Improved Solution for a Post-Pandemic Informative System: A Ref...

\subsection{Data sourcing}

Our research approach is based on analytical calculations and simulations. Sourcing accurate data about natural learners seems complicated as the results require a significant number of simulated learners. This requirement hinges on this simulation's realworld application because of the restrictive nature to access multi-content and multidimensional learners. This complexity of access and the applicable situation that demands human resources and financial support also limit the application in the real world [9].

Many variables can affect the learners' outcomes, and real-world applications can not see all data about natural learners. Opting for virtual simulations of this suggested model and produce statistical analysis is more achievable. Using computer-based recreation and then statistically analyze the outcomes will be more truthful to reality [9].

Furthermore, a random generator is needed to simulate score results. This generator is changeable depending on the researcher's preferable generator. However, the results should not depend on the generator. The results of the simulation should be reproducible. His scores trace the learner's path as they continue in an educational environment modeled after a random walk. The record of every level up or down will gradually build a list of data points of each learner path; multiplying by the numbers of simulated learners can clarify the overall trend [9].

In each simulation, results should index and routine iterations over the path of the learner. The results then are appended to the list of records. However, the score's observations should be based on previous appended records to keep the paths personalized and unique to the simulated learner. The reader should keep in viewpoint the script's goal to maximize the acquisition of learning and knowledge based on the procurement of data and information weekly.

\subsection{Analytics tools}

In addressing the simulation's complexity, this paper selects Python as the computer language to simulate it. This situation is intricate, and many variables can affect the outcome. Producing an analytical solution can be considered achievable, but it will need accurate data for adjusting it [9]. Python has many advantages as it is a general-purpose programming language, easy to read, write and reproduce, making it a popular solution for data analytics. It also has better community support because of its open-source nature. Furthermore, Python has more extensions into other fields and applications.

There is also the $\mathrm{R}$ language that is built specifically for data analysis and visualizations. It is popular among general data scientists, especially with a statistical background. It offers rapid prototyping of data-driven applications and dashboards. However, it is not selected for this simulation that does not mean its dismissal. SQL is the dominant solution in a real-world application to design and manage data held in relational database systems. Python allows the import, cleaning, manipulating, and visualizing of data [9]. 
Paper-Simple Technology is an Improved Solution for a Post-Pandemic Informative System: A Ref...

\subsection{Ethical considerations}

Deepen in the study; some ethical warnings should portray for the actual application of the simulation. With the increased use of technology, users tend to overlook moral aspects of the exploration, which ignore the data sharing protocols, cultural, social, and economic complexities of the organization and learners, generalizations in seeking results. Likewise, the actors' consent is primordial and always needed because they can explain their data more than an expert analyst. Concerning the actors' privacy, the future endeavor should consult on the security protocols towards using data for the actors' profit, not against them [10].

Additionally, building on previous studies is recommended to avoid the mistakes of past applications. Skeptic and critical thinking should constantly be introduced when handling data. Practitioners ought to review their acts of practices and approaches to question their actions if they are for the learners' benefit. A governance plan and leadership are also crucial for ethical practices and applications. Without them, it is hard to navigate divided from guidance. In applying technology, the focus shifts toward the features and makes activities efficient in attaining goals and effectively using a reduced number of efforts. The focus should be on understanding and adapting to the actors' needs with an open set of mindfulness and acceptance [10].

\section{Results}

In the following, a rigorous description of the resulted simulation script is provided with a notation of the required packages for the simulation.

The first outcome, is simulating a learner. For this goal, a random seed needed for the reproducibility and checking of the results.

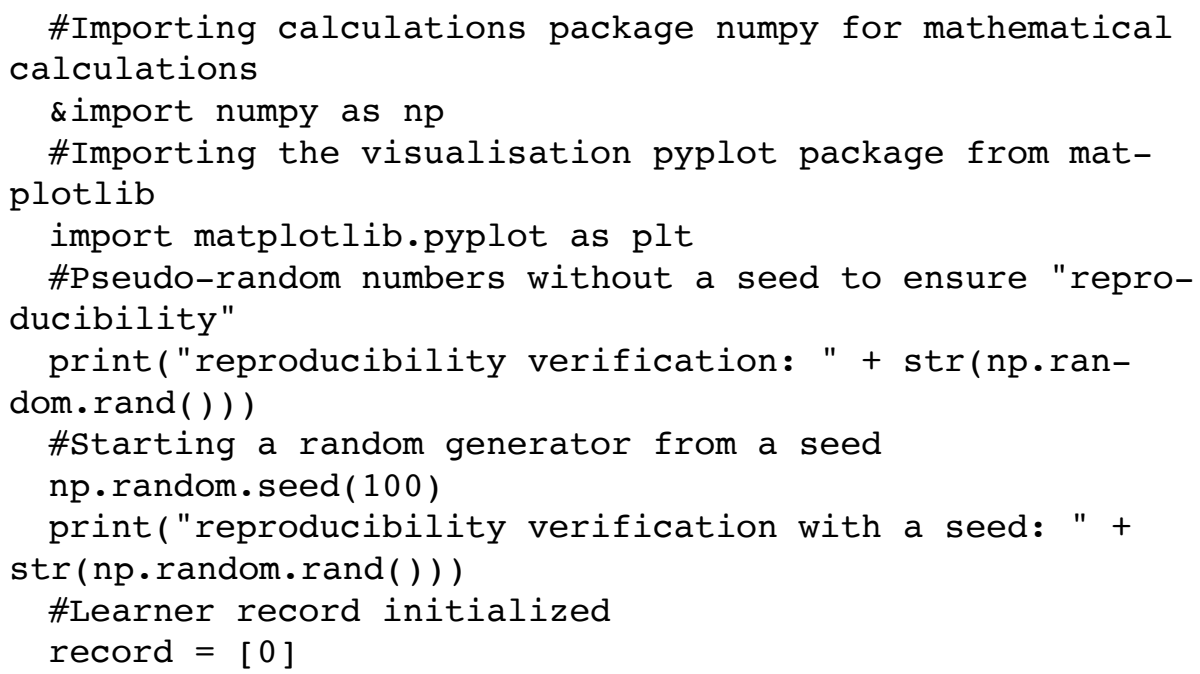




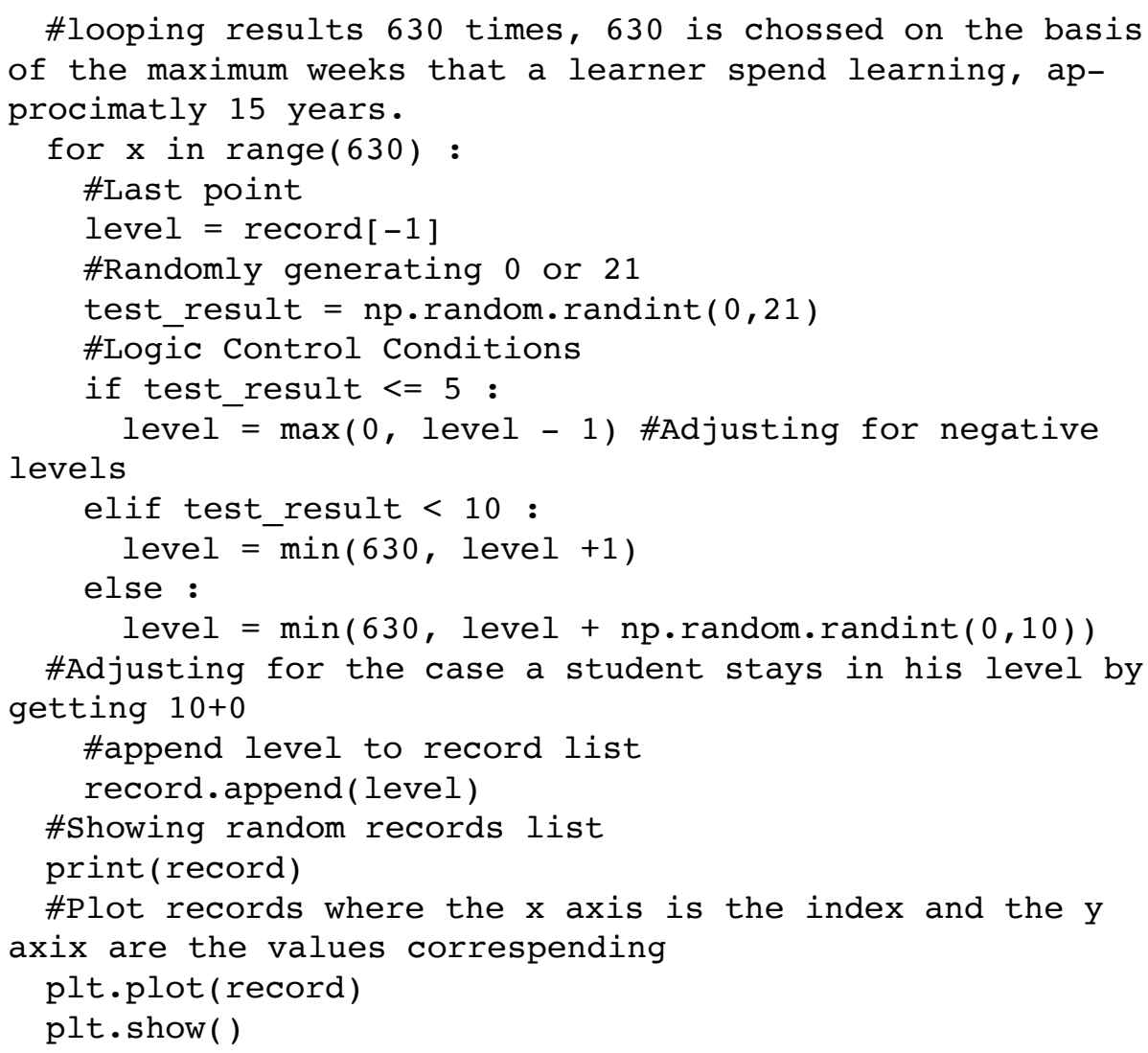

The script produce a random walk in the list of records and a line visualization.

\subsection{Closing the gap}

However, it does not answer the main request of optimizing the learner level to reach above $90 \%$ success rate. Tough the script, observations were noted:

- Each record generated has a different end level.

- Multiplying the students will produce a range of endpoints.

- The distribution of levels is the result of simulating the endpoints.

- Identifying distribution is critical to calculating the chances of reaching the identified aim.

As a solution, a list is needed to save the last records of all simulated learners. All the values represent a distribution that histograms can visualize. The generated histogram representing the distribution should converge to a bell shape to confirm the theoretical distribution, which means that the distribution follows ordinary law's mathematical calculation.

In consequence, the following script gives an impression about the correct numbers of tests. 


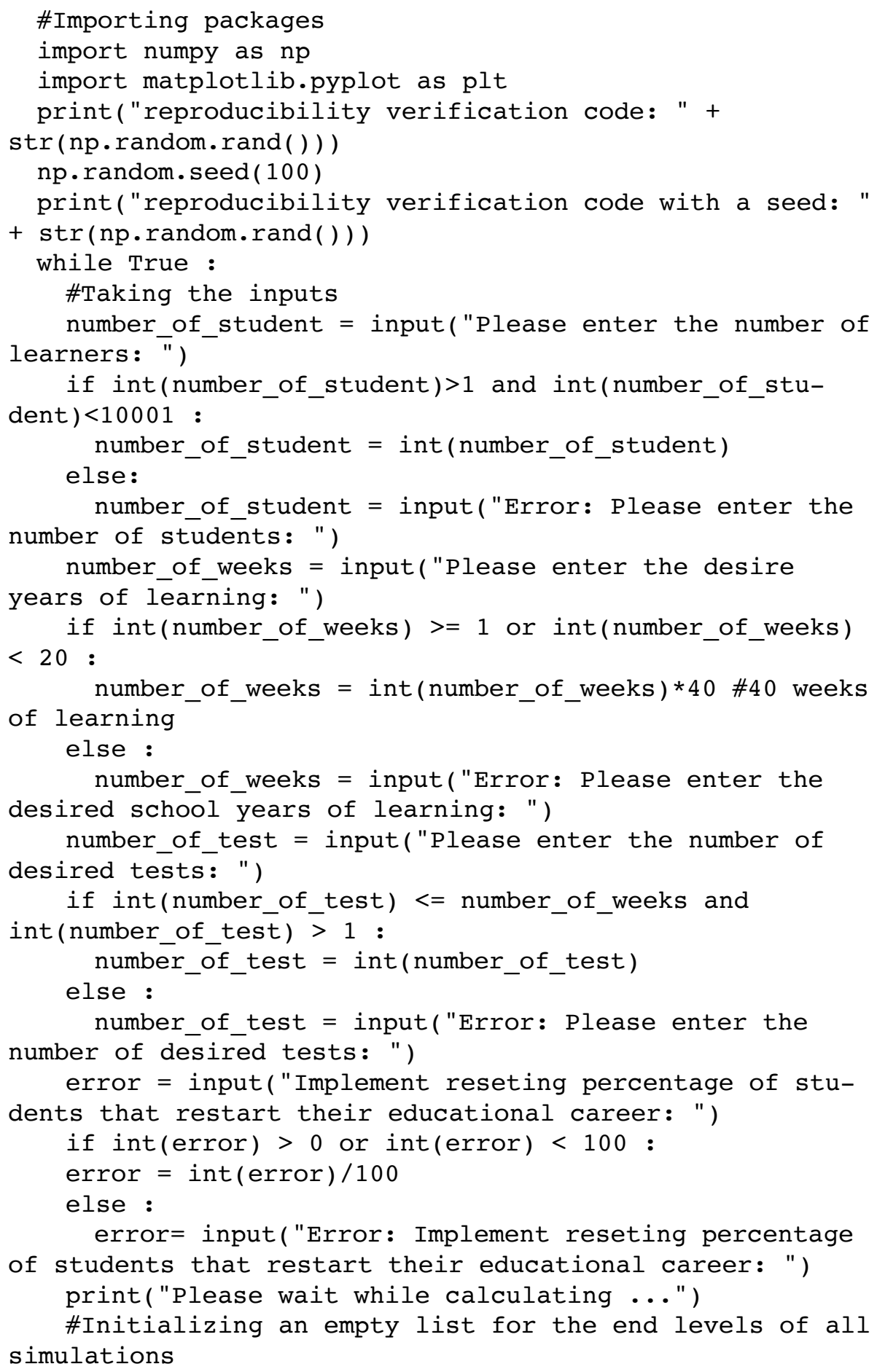




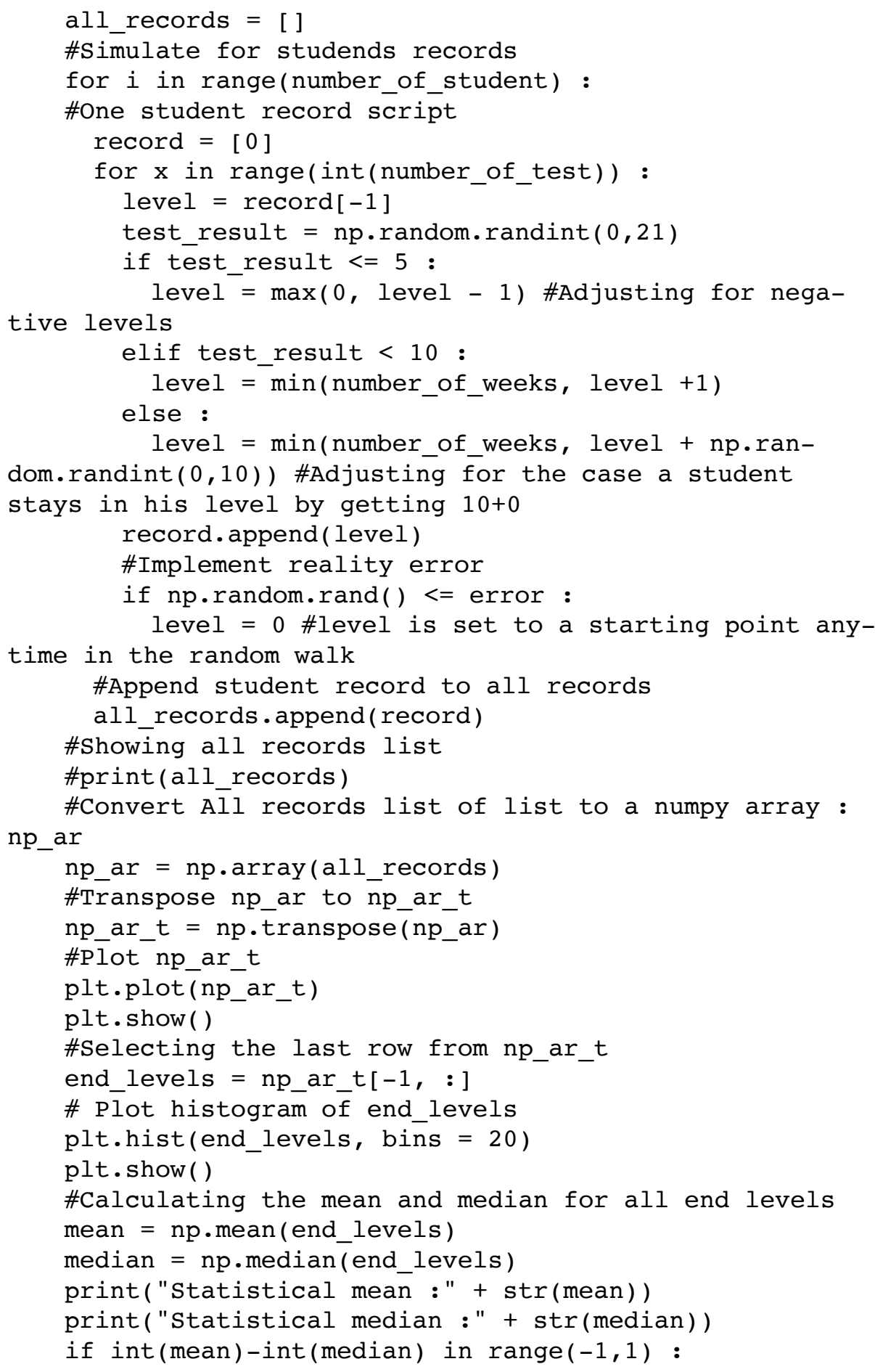




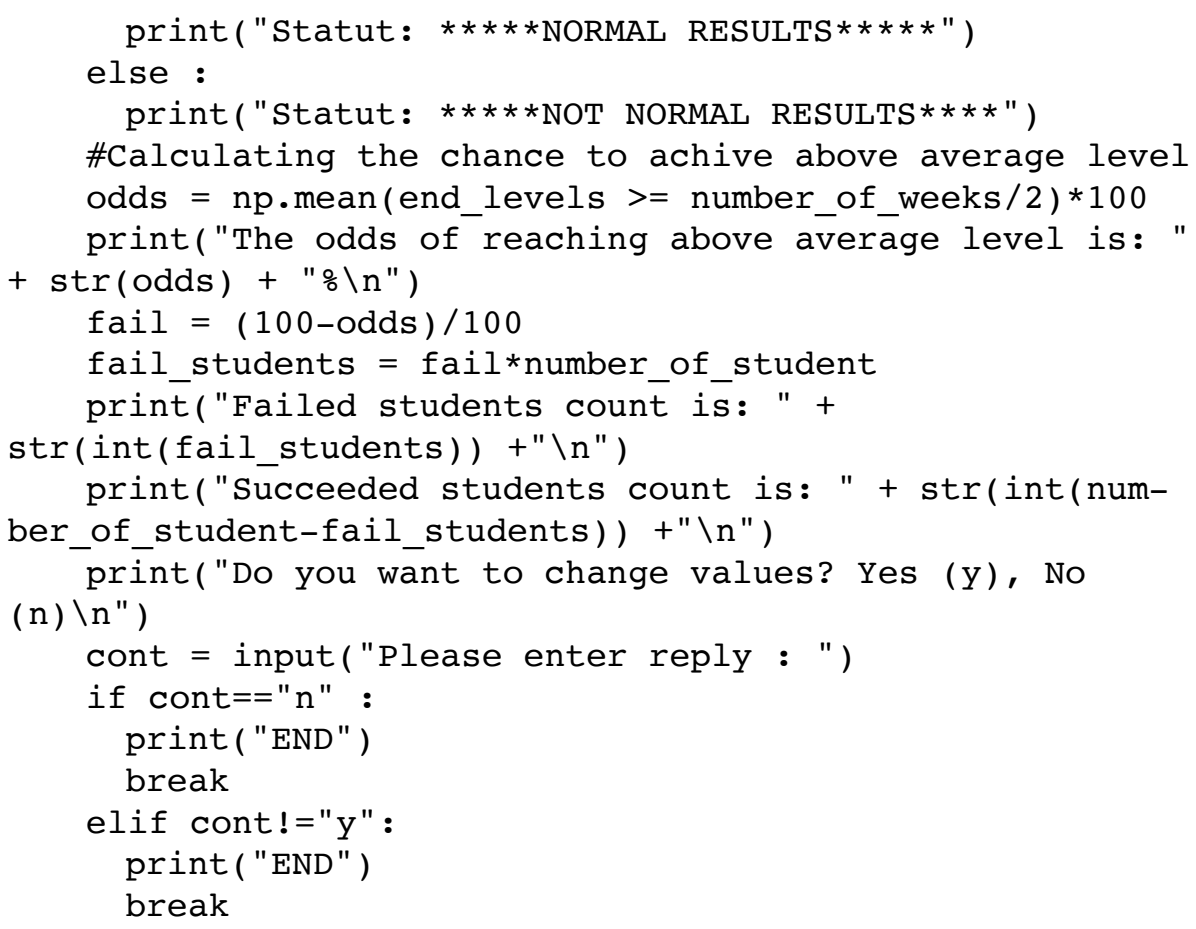

\subsection{Emerging facts and patterns}

Executing the script, input informations are choosed by the desciders to design the number of tests to reach success and see the number of failed learners to design adequate support systems.

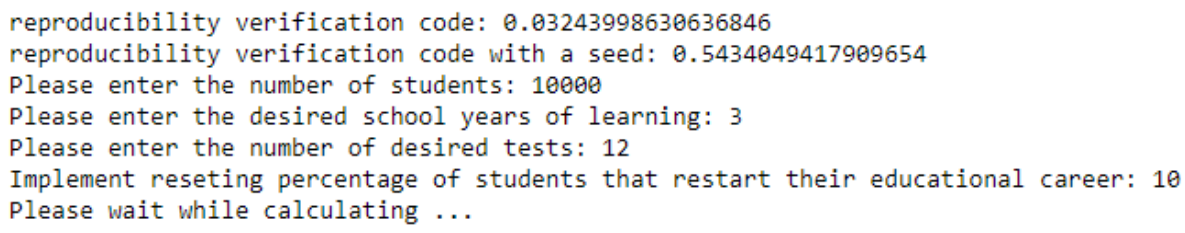

Fig. 1. Illustration of input data 


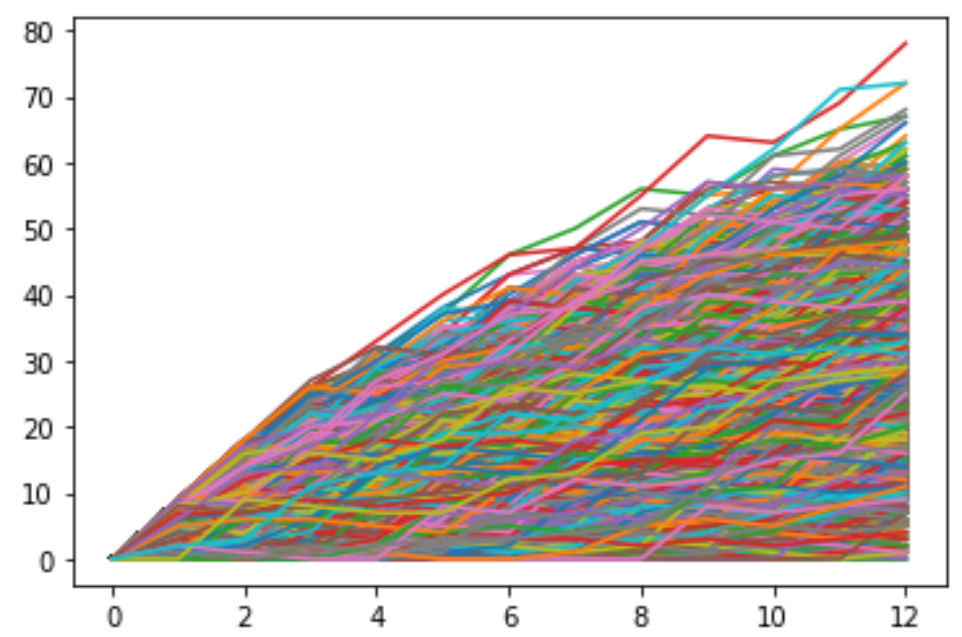

Fig. 2. Visualization of the different learning paths

Each line represents the advancement of a particular simulated learner. Some learners lose their momentum while others keep it forward. However, this visualization does not show if the results are typical or not; therefore, a histogram distribution of the learners' population with their achieved results is needed to check the mathematical accuracy of the simulation. The statistical analysis related to histograms indicates if the Data is unimodal or not. The meaning of it resides in checking if the learner's data follow a single-mode identified by a single peak.

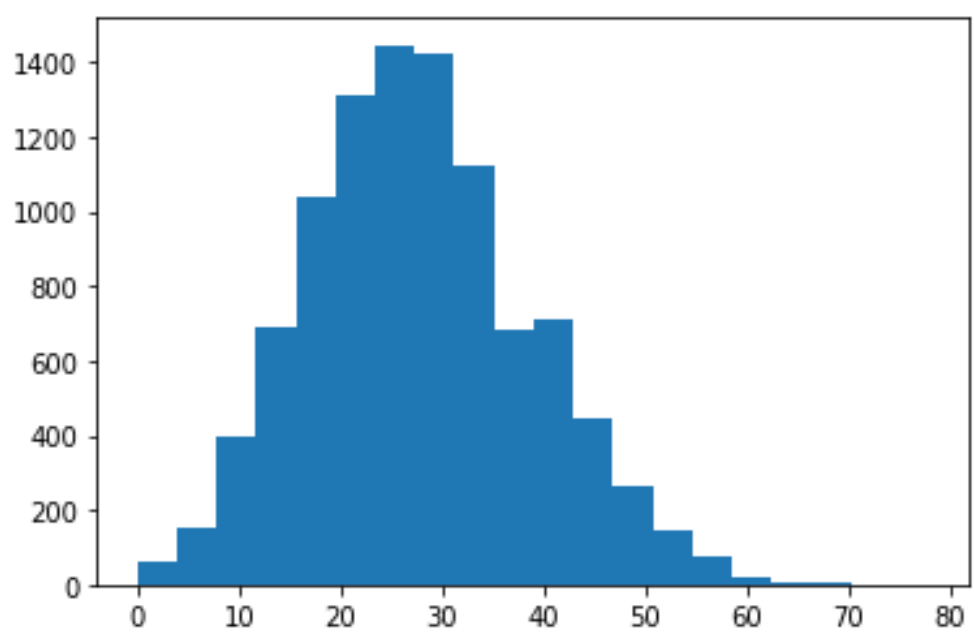

Fig. 3. Statistical visualization of the distribution of learners levels 


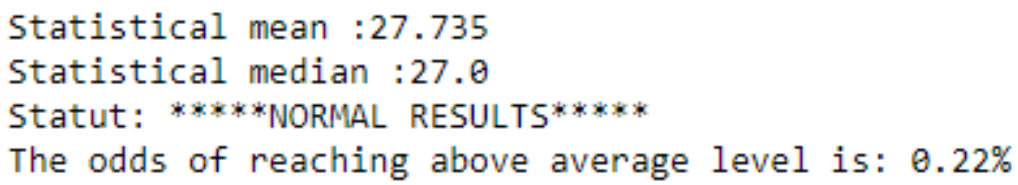

Fig. 4. Illustration of output data

\section{Discussion}

Crisscrossing understanding of the results, the Table 1 show results for the same count of the students, which is fixed in 10000 (With a 10\% dropout rate) to cover typical learners count in the context of this study location.

The test numbers are based on the average tests in the curriculum's sub-module, which accounts for the control test and final exam. Our model suggests that the eight years curriculum can be shortened into four years curriculum with the aid of a technological simplification and identification of essential data points in the curriculum with their links. On the basis of the locally studied curriculum, the Table 1 numbers represent an approachable estimate of the entire curriculum. However, deepen the analysis is required by the reader to check the results of this manuscript on the basis of their curriculum design and instructions [11].

Table 1. Simulation outputs

\begin{tabular}{|c|c|c|c|c|c|}
\hline Type Teaching/Learning & Years & Tests & Average & Odds* & Description \\
\hline \multirow{3}{*}{$\begin{array}{l}\text { Traditional (Tests are based on the } \\
\text { objectives of the curriculum) }\end{array}$} & 3 & 72 & \multirow{6}{*}{$50 \%$} & $100 \%$ & \multirow{2}{*}{ Not normal results } \\
\hline & 5 & 108 & & $100 \%$ & \\
\hline & 8 & 118 & & $99.93 \%$ & \multirow{6}{*}{ Normal results } \\
\hline \multirow{3}{*}{$\begin{array}{l}\text { Digital (Tests are based on the re- } \\
\text { tention of the curriculum content, } \\
\text { which maximizes the count in tra- } \\
\text { ditional setup) }\end{array}$} & 3 & 96 & & $100 \%$ & \\
\hline & 5 & 130 & & $100 \%$ & \\
\hline & 8 & 132 & & $99.97 \%$ & \\
\hline \multirow{3}{*}{$\begin{array}{l}\text { Proposed Hybrid Model } \\
\text { (Tests are basic and weekly) }\end{array}$} & \multirow{3}{*}{4} & 148 & $60 \%$ & $100 \%$ & \\
\hline & & 168 & $70 \%$ & $100 \%$ & \\
\hline & & 208 & $80 \%$ & Error & Too many tests \\
\hline
\end{tabular}

The simulation outputs in Table 1 show the odds of reaching an above-average level. Based on simulated data results. It becomes evident that to reach above-average learning retention results, and it is primordial to design technologies that incorporate essential data points for the learners to reach weekly and diversify the learning environment to use these data points to become information pieces and knowledge. The crossbreeding between traditional and digital instructional impose forward strategical thinking on designing new technologies for learning that employ basic data-points of knowledge and information as a source of building successful retention. 
However, by simplifying processes and based decisions on data, organizations can reduce investments in new hardware to adverse possessions and expand their life cycle performances in educational technologies. For example, organizations can rely on "Quizzing systems" to test 20 essential data points about the weekly curriculum. Therefore, opting to enhance the quizzing technology by incorporating more collection of passive data about the learners. Furthermore, future activities necessitate producing curriculums based on the model described in the results section and include more existing technology with effective implementation models.

The well-established tradition of teaching and learning incorporated and confined into a specific space was not an option as the population's growth made it unsustainable, especially in the limitation imposed for social distancing required to limit the population's mobility quarantine purposes. Digital spaces and physical spaces are intertwined, and they are both impacted homogenously. Data is the everyday reality point. Studying data in educational setup refers us to Learning Analytics (L.A.) as a helpful proposition for solving the current issues mainly caused by the urgent rise of demand and restricted supply chain. By extracting intelligence from data for the sole purpose of understanding and improving learning practices in physical and digital spaces, this paper anticipates the present solution to the paradigm of supply and demand [12].

In any case, the flood of assorted advancements and structures mentioned put pressure on the deciders to pick the best way of embracing the new situations without losing proficiency and viability of their very much confident traditionality. The reference model and simulations provided work parallel with what exists hence easing the burden on deciders [12].

\section{Conclusions}

The study's orientation is not closed to a single discipline, and it encourages an interdisciplinary verge to assess the usefulness of the brought-out ideas in this study. This paper presents a cornerstone to propose new ways to develop, implement, integrate, and regulate educational technologies into the instructional organization to strengthen these organizations' types into facing future challenges as the unities presented by the covid-19 pandemic. In conclusion, our reference model developed based on simulated data requires identifying data points of knowledge in the curriculum and their relation to test learner's weekly retention and use of them. However, it would be a mess judgment if the converged thinking predicts that this is the only way to adapt and improve the instructional system. The developing new technologies for learning constructs an opportunity to address the lack of data-based decision-making inside educational organizations. This manuscript can promote further progress in the sphere of technological development. However, innovative pathways should be provided to the moral principles shared by the academic and scientific communities worldwide.

This paper was limited by access to funds and resources to simulate the accurate actual model. Nevertheless, it still constructs junctures for other researchers to apply and test the results of this paper. 
Paper - Simple Technology is an Improved Solution for a Post-Pandemic Informative System: A Ref...

\section{References}

[1] Ferguson, R. (2012). Learning analytics: drivers, developments, and challenges. International Journal of Technology Enhanced Learning, 4(5/6), 304. https://doi.org/10.1504/ijtel. 2012.051816

[2] Chatti, M. A., Dyckhoff, A. L., Schroeder, U., \& Thüs, H. (2012). A reference model for learning analytics. International Journal of Technology Enhanced Learning, 4(5/6), 318. https://doi.org/10.1504/ijtel.2012.051815

[3] Gašević, D., Dawson, S., Rogers, T., \& Gasevic, D. (2016). Learning analytics should not promote one size fits all: The effects of instructional conditions in predicting academic success. The Internet and Higher Education, 28, 68-84. https://doi.org/10.1016/j.iheduc.2015.10.002

[4] Kizilcec, R. F., Pérez-Sanagustín, M., \& Maldonado, J. J. (2017). Self-regulated learning strategies predict learner behavior and goal attainment in Massive Open Online Courses. Computers \& Education, 104, 18-33. https://doi.org/10.1016/j.compedu.2016.10.001

[5] Housni, M., Namir, A., Talbi, M., \& Chafiq, N. (2018). Applying Data Analytics and Cumulative Accuracy Profile (CAP) Approach in Real-Time Maintenance of Instructional Design Models. Advances in Intelligent Systems and Computing, 17-25. https://doi.org/10.1007/978$\underline{3-319-91337-7 \quad 2}$

[6] Belarbi, N., Chafiq, N., Talbi, M., Namir, A., \& Benlahmar, E. (2019). User Profiling in a SPOC: A method based on User Video Clickstream Analysis. International Journal of Emerging Technologies in Learning (IJET), 14(01), 110. https://doi.org/10.3991/ijet.v14i01.9091

[7] Tempelaar, D. T., Rienties, B., \& Giesbers, B. (2015). In search for the most informative data for feedback generation: Learning analytics in a data-rich context. Computers in Human Behavior, 47, 157-167. https://doi.org/10.1016/j.chb.2014.05.038

[8] Berland, M., Martin, T., Benton, T., Petrick Smith, C., \& Davis, D. (2013). Using Learning Analytics to Understand the Learning Pathways of Novice Programmers. Journal of the Learning Sciences, 22(4), 564-599. https://doi.org/10.1080/10508406.2013.836655

[9] Pardo, A., Jovanovic, J., Dawson, S., Gašević, D., \& Mirriahi, N. (2017). Using learning analytics to scale the provision of personalized feedback. British Journal of Educational Technology, 50(1), 128-138. https://doi.org/10.1111/bjet.12592

[10] Ferguson, R. (2019). Ethical Challenges for Learning Analytics. Journal of Learning Analytics, 6(3), 25-30. https://doi.org/10.18608/jla.2019.63.5

[11] El-Sofany, H. F., El-Seoud, S. A., Ghaleb, F. F. M., Ibrahim, S., \& Al-Jaidah, N. (2009). Questions-Bank System to Enhance E-Learning in School Education. International Journal of Emerging Technologies in Learning (IJET), 4(3), 8. https://doi.org/10.3991/ijet.v4i3.978

[12] Schweighofer, J., Taraghi, B., \& Ebner, M. (2019). Development of a Quiz - Implementation of a (Self-) Assessment Tool and its Integration in Moodle. International Journal of Emerging Technologies in Learning (IJET), 14(23), 141. https://doi.org/10.3991/ijet.v14i23.11484

\section{$7 \quad$ Authors}

Mohamed Housni is a teacher of Mathematics at the Ministry of National Education in Morrocco, Regional Academy of Education and Training Casablanca-Settat (AREF Casa-Settat). He is Also a Ph.D. candidate at the Laboratory of Information Technologies and Modelling (LTIM), Department of Mathematics and Computer science-Fac- 
ulty of Sciences Ben M'Sick-University HASSAN II, B.P 7955 Sidi Othmane, Casablanca, Morocco. His area of research focuses on applying Learning Analytics in the Moroccan Educational System.

Mohammed Talbi is the Dean of the Faculty of Sciences Ben M'Sick and President of the Observatory of Research in Didactics and University Pedagogy (ORDIPU). He has a Ph.D. in Chemistry from the University Pierre et Marie Curie, Paris, France, and a member of the Multidisciplinary Laboratory in Sciences and Information, Communication, and Educational Technology (LAPSTICE), Departement of Communication, Faculty of Sciences Ben M'Sick, University HASSAN II, B.P 7955 Sidi Othmane, Casablanca, Morocco. His research aims to improve the educational environment.

Abdelwahed NAMIR is the vice-Dean at the Faculty of Sciences Ben M'Sik and a professor of mathematics and computer sciences at the same faculty. He has a Ph.D. in Numerical Methods for Engineers from Mohammadia School of Engineers (EMI Rabat) Morocco. He is also a founding member of the Laboratory of Information Technologies and Modelling (LTIM), Department of Mathematics and Computer scienceFaculty of Sciences Ben M'Sick-University HASSAN II, B.P 7955 Sidi Othmane, Casablanca, Morocco. His area of research focuses on the modelization of reality using computer science methods and mathematics.

Article submitted 2021-04-10. Resubmitted 2021-05-03. Final acceptance 2021-05-17. Final version published as submitted by the authors. 